\title{
NHC-Boranes: Air- and Water-tolerant Co- initiators for Type II Photopolymerizations
}

\author{
Emmanuel Lacôte ${ }^{\star a}$, Dennis P. Curran ${ }^{b}$, and Jacques Lalevée ${ }^{\star_{c}}$
}

\begin{abstract}
N}$-Hetereocyclic carbene $(\mathrm{NHC})$ complexes of boranes are stable compounds that can be used as coinitiators for the type II photopolymerization of acrylates. The present account summarizes this new development for polymerization and boron chemistry. In particular, NHC-boranes are air- and water-tolerant, which enhances the practicality of the photopolymerizations.
\end{abstract}

Keywords: Boron · Carbenes · Free radicals · NHC-Boranes · Photopolymerization

\section{Introduction}

Radical photopolymerizations are essential for some industrial applications. They are used in laser imaging and radiation curing in applications such as inks and coatings. Radical photopolymerizations start from irradiation of a photoinitiating system (PI). The radicals that are formed initiate crosslinking polymerization. ${ }^{11]}$ There are two distinct types of PIs (Scheme 1). In type I systems, light irradiation directly generates radicals through homolysis of a suitable bond. Type II systems first produce the excited triplet state of the initiator (e.g. benzophenone). The excited state abstracts a hydrogen atom from another molecule (the co-initiator), delivering radicals that can initiate the polymerization.
NHC-boranes are formed from complexation of boranes with N-heterocyclic carbenes. They form a new family of reagents for organic synthesis. ${ }^{[2]}$ We have discovered that $\mathrm{NHC}$-boranes easily give the corresponding NHC-boryl radicals through hydrogen abstraction by alkoxyl radicals. ${ }^{[3]}$ We surmised that this would make them good co-initiators for type II photopolymerization because the triplet state of benzophenone obtained through irradiation behaves similarly to alkoxyl radicals. In the present account, we review the reactivity of NHC-boranes as co-initiators for type II photopolymerization of acrylates.

Type I
Type II

${ }^{\star}$ Correspondence: Dr. E. Lacôte ${ }^{a}$; Prof. Jacques Lalevée ${ }^{\mathrm{c}}$

Tel.: +3316982 3116; +33 389608803

E-mail: emmanuel.lacote@icsn.cnrs-gif.fr; jacques.lalevée@uha.fr

aCSN CNRS

Av. de la Terrasse

F-91198 Gif s/Yvette Cedex, France

bUniversity of Pittsburgh

Department of Chemistry

Pittsburgh, PA 15260, USA

IS2M - LRC CNRS 7228

ENSCMu-UHA

15 rue Jean Starcky

F-68057 Mulhouse Cedex, France

\section{Physical Organic Basis for NHC- Boryl-initiated Polymerizations}

The formation of the NHC-boryl radicals can be easily established by ESR-spin trapping experiments (Fig. 1). A nitroxide radical is obtained by addition of radicals onto phenyl-N-tert-butylnitrone (PBN), whose ESR spectrum can be recorded. For example, irradiation of $\mathbf{8}$ (see structures in Table 1) in the presence of di-tert-butylperoxide and PBN leads to the expected spin adduct of the NHC-boryl radical, characterized by $\mathrm{a}_{\mathrm{N}} \sim 15.4 ; \mathrm{a}_{\mathrm{H}} \sim 2.1 \mathrm{G}$ and a third hyperfine coupling $\left(\mathrm{a}_{\mathrm{B}} \sim 4.4 \mathrm{G}\right)$ directly associated with the presence of a boron nucleus. ${ }^{[3]}$

Through the observation of NHCboryls in laser flash photolysis, the reaction rate constants for the polymerization elementary steps, reactions (1)-(3) (where $\mathrm{BP}$ is benzophenone and $\mathrm{BPH}^{\bullet}$ is $\mathrm{Ph}_{2} \mathrm{CH}\left({ }^{\circ}\right)$ $\mathrm{OH}$ ), can be easily determined (Table 1).

Scheme 1. The two types of radical photopolymerization.

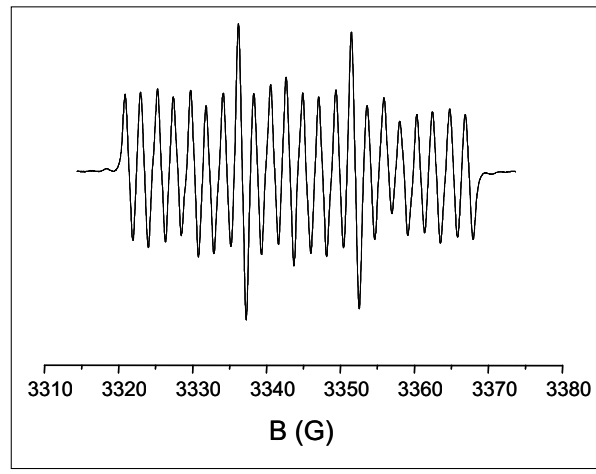

Fig. 1. ESR spectrum observed for the adduct of the NHC-boryl radical derived from $\mathbf{8}$ in tertbutylbenzene/di-tert-butylperoxide with PBN.

\footnotetext{
$t-\mathrm{BuO}^{\bullet}+\mathrm{NHC}-\mathrm{BH}_{3} \rightarrow \mathrm{tBuOH}+\mathrm{NHC}-\mathrm{BH}_{2}{ }^{\circ}$

${ }^{3} \mathrm{BP}+\mathrm{NHC}-\mathrm{BH}_{3} \rightarrow \mathrm{BPH}^{\cdot}+\mathrm{NHC}_{-} \mathrm{BH}_{2}$

$\mathrm{NHC}-\mathrm{BH}_{2} \cdot+\mathrm{CH}_{2}=\mathrm{CH}-\mathrm{CO}_{2} \mathrm{Me} \rightarrow \mathrm{NHC}^{2}-\mathrm{BH}_{2}-\mathrm{CH}_{2} \mathrm{CH}\left({ }^{\circ}\right) \mathrm{CO}_{2} \mathrm{Me}$
} 
Comparison of rate constants for reactions (1) and (2) ( $k_{1}$ and $\left.k_{2}\right)$ would confirm our initial assumption that the triplet state of benzophenone could lead to NHC-boryl radicals through $\mathrm{H}$ atom abstraction, while the rate constant for the addition of NHCboryl radicals to the monomer model methyl acrylate $\left(k_{a d d}\right)$ is a crucial data to know, since this is the initiation of the polymerization. A high $k_{\text {add }}$ value is required for efficient radical polymerization.

High rate constants were found for the formation of NHC-boryl radicals $\left(k_{1}, k_{2}\right)$, typically in the $10^{6}-10^{7} \mathrm{M}^{-1} \mathrm{~s}^{-1}$ range. ${ }^{[4,5]}$ Furthermore, the high reactivity of NHCboryl radicals toward electron-poor olefins means the NHC-boryls are very nucleophilic. The rate constants $\left(k_{\text {add }}\right)$ are higher than those derived from well known polymerization initiating radicals, including the aminoalkyl radicals derived from ethyldimethylaminobenzoate (EDB) or the benzoyl radicals, whose $k_{\text {add }}$ are $5.0 \times 10^{5}$ and $2.7 \times 10^{5} \mathrm{M}^{-1} \mathrm{~s}^{-1}$, respectively. ${ }^{[1]} \mathrm{NHC}$ borane 4 is water-soluble. ${ }^{[6]}$ Interestingly, its rate constants were not affected by the presence of water (see below). ${ }^{[7]}$

\section{Photopolymerization of Acrylates with NHC-Borane Co-initiators}

The cross-linking polymerization kinetics of different acrylates (trimethylolpropane triacrylate, TMPTA and ethoxylated pentaerythritol tetraacrylate, EPT) were examined by measuring the consumption of monomer in different type II photoinitiating systems containing NHC-boranes. ${ }^{[5]}$ Benzophenone was used as photoinitiator to ensure good light absorption properties in the 300-350 $\mathrm{nm}$ range (Scheme 2)

Two kinds of photopolymerization conditions were examined. In open conditions, films are deposited on a pellet and irradiated. During the process, oxygen from the air can diffuse into the formulation. Alternatively, in laminated conditions the photosensitive formulation is sandwiched between two polypropylene films to ensure that no oxygen can enter the medium beyond that initially dissolved.

In the absence of NHC-boranes, the polymerization is negligible in open conditions and a high inhibition time was found in laminate. Oxygen can quench the excited state of BP, and trap the various radicals involved in the polymerizations, from the initiating species to the radicals on the growing polymer chains (Scheme 3). A stronger inhibition is expected for polymerization under air since continuous diffusion of oxygen can keep shutting down polymerization, whereas in laminate there is no oxygen once the amount initially present is consumed. In open conditions, inhibition can be very persistent (Fig. 2,

Table 1. Rate constants $\left(k_{1}, k_{2}\right)$ for the formation of the NHC-boryl radicals and rate constants of addition of the generated NHC-boryls onto methylacrylate (MA).

\begin{tabular}{|c|c|c|c|c|}
\hline Entry & NHC-borane & $\begin{array}{l}\mathrm{k}^{1 \mathrm{a}}(t \mathrm{Bu}-\mathrm{O} \bullet) \\
\mathrm{x} 10^{7} \mathrm{M}^{-1} \mathrm{~s}^{-1}\end{array}$ & $\begin{array}{l}\mathrm{k}^{2}\left({ }^{3} \mathrm{BP}\right) \\
\mathrm{x} 10^{7} \mathrm{M}^{-1} \mathrm{~s}^{-1}\end{array}$ & $\begin{array}{l}\mathrm{k}_{\text {add }}^{\mathrm{a}}(\mathrm{NHCB} \bullet / \mathrm{MA}) \\
\times 10^{6} \mathrm{M}^{-1} \mathrm{~s}^{-1}\end{array}$ \\
\hline 1 & $\mathrm{Ph}$ & 15 & $55\left(0.9^{b}\right)$ & 6.0 \\
\hline 2 & & 9.5 & $28\left(0.25^{\mathrm{b}}\right)$ & 0.08 \\
\hline 3 & & 12 & $39\left(0.55^{\mathrm{b}}\right)$ & 0.52 \\
\hline 4 & & 14 & nd & 17 \\
\hline 5 & & 24 & $120\left(0.7^{b}\right)$ & 35 \\
\hline 6 & & 11 & $250\left(<0.15^{b}\right)$ & 9.5 \\
\hline 7 & & 26 & $45.5\left(1^{b}\right)$ & 31 \\
\hline 8 & & 17 & 68.7 & 1.9 \\
\hline 9 & & 26 & 96 & 38 \\
\hline 10 & & 14 & 91.1 & 11 \\
\hline 11 & & 21 & $100\left(1^{b}\right)$ & 21 \\
\hline 12 & & 26.2 & nd & 10.5 \\
\hline
\end{tabular}

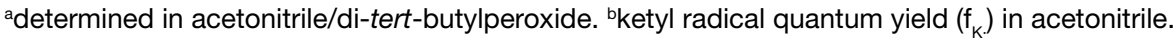

bottom, curve a for a typical example with NHC-borane 7), while in laminate conditions inhibition periods are generally observed before polymerization sets in (Fig. 2, top, curve a for an example with NHCborane 6 ).

In both conditions the polymerizations generally start immediately and give higher conversions when NHC-boranes are present.

Air-tolerant radical polymerizations are important from a practical point of view because they do not require oxygen exclusion and the polymer could be grown directly on the desired surfaces and objects. We thus investigated the mechanism 


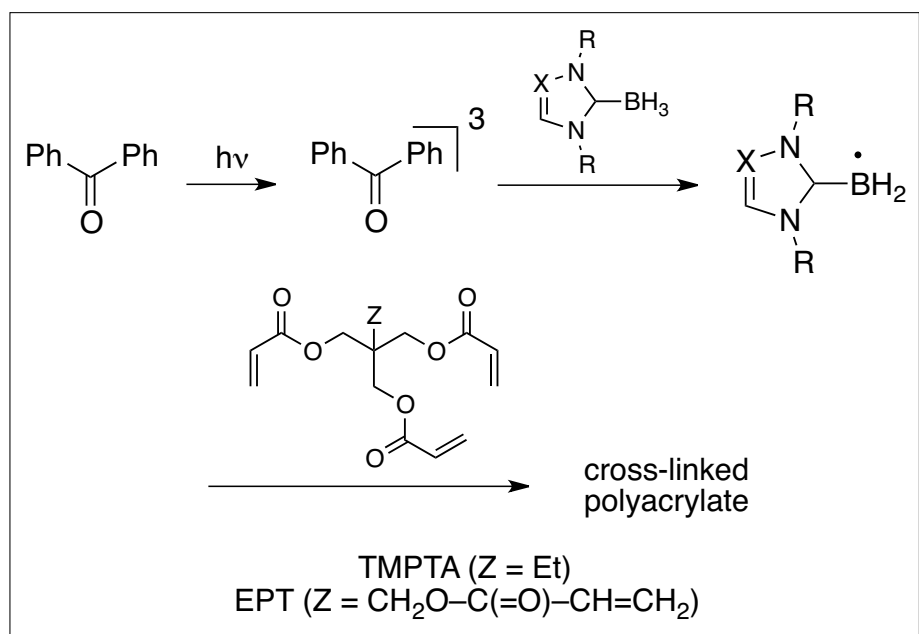

Scheme 2. Use of NHC-boranes as type II photopolymerization co-initiators.

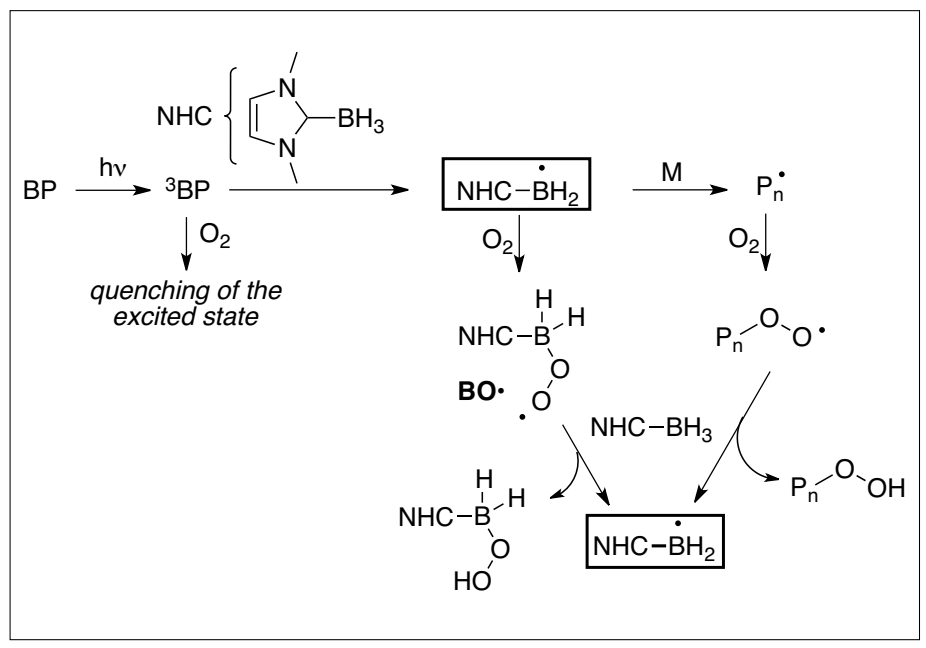

Scheme 3. Proposed mechanism for overcoming oxygen inhibition by NHC-boranes.

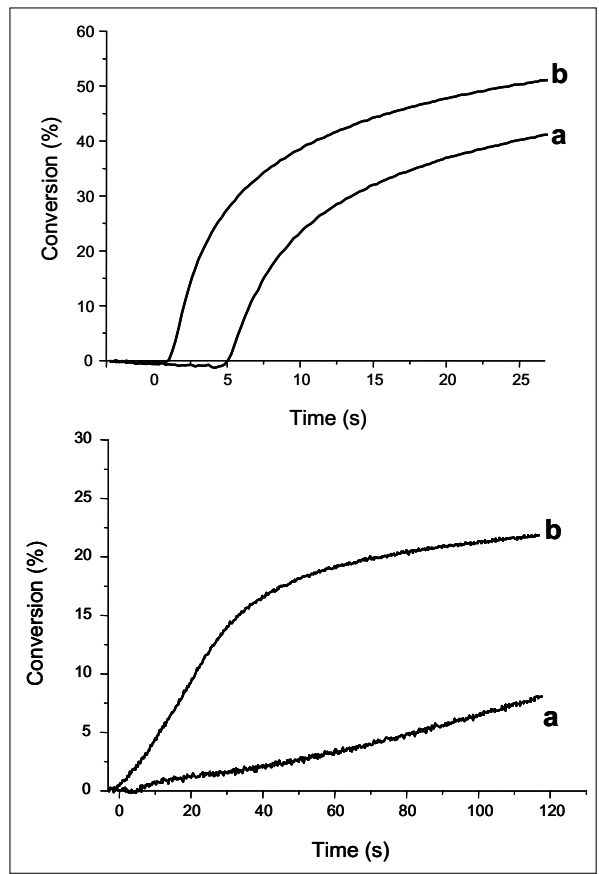

Fig. 2. Radical photopolymerization ability of $\mathrm{BP} / \mathrm{co}$-initiator couples (1\%/1\% w/w; in EPT; $\lambda>300 \mathrm{~nm}$ ). In laminate (top): (a) only BP; (b) BP/6. Under air (bottom): (a) BP/ethyldimethylaminobenzoate; (b) BP/7.

for overcoming the inhibition (Scheme 3). ${ }^{[4]}$

Oxygen can react rapidly with NHC$\mathrm{BH}_{2} \cdot$ to give bora-peroxyl radical $\mathbf{B O}^{*}{ }^{[5]}$ Intermediate $\mathbf{B O}^{*}$ reacts fast with $\mathrm{NHC}$ $\mathrm{BH}_{3}$ to regenerate the initiating NHC-boryl radical. ${ }^{[4]}$ On the other hand, the electrophilic peroxyl radicals created by trapping of the radicals on the growing polymer chains with oxygen react rapidly with the polarity matched nucleophilic NHCboranes, again regenerating the initiating NHC-boryl radicals. This turnover maintains efficient initiation.

The additional nitrogen atom in NHCborane 4 makes this compound very soluble in water. ${ }^{[6]}$ As a consequence we extended the NHC-borane-coinitiated type II photopolymerizations to water-soluble monomers. ${ }^{[7]}$ For example, irradiation of an aqueous solution of hydroxyethyl acrylate (HEA) in the presence of $\mathbf{4}$ and the water-soluble initiator 1-chloro-4-hydroxy thioxanthone led to the formation of PHEA $\left(\mathrm{M}_{\mathrm{n}}=3300 \mathrm{~g} \cdot \mathrm{mol}^{-1}\right.$, PDI = 3.3, Fig. 3). As with the other monomers, HEA could not be photopolymerized without the twocomponent system.

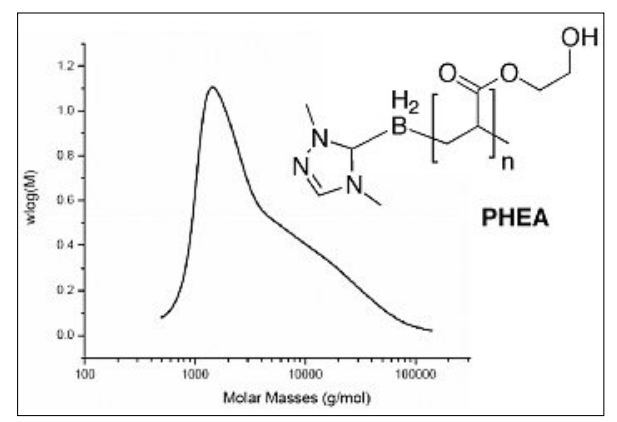

Fig. 3. Size-exclusion chromatography (SEC) analysis of the PHEA synthetized from HEA with $4(3 \% \mathrm{w} / \mathrm{w})$ and 1-chloro-4-hydroxy thioxanthone $(0.1 \%)$.

\section{Acceleration of the Photopolymerizations with Diphenyliodonium}

The addition of diphenyliodonium hexafluorophosphate $\left(\mathrm{Ph}_{2} \mathrm{I}^{+} \mathrm{PF}_{6}^{-}\right)$to the PI improves the polymerization of acrylates. The iodonium salt oxidizes the radicals obtained from the co-initiator, delivering the extremely reactive phenyl radicals, whose fast addition to acrylates leads to an improved initiation (Scheme 4).

One sterically hindered NHC-boryl radical was oxidized electrochemically to the corresponding borenium. ${ }^{8]} \mathrm{We}$ showed that the NHC-boryl radicals were chemically oxidized to the boreniums at a rate close to diffusion control. ${ }^{[4,5]}$ As a consequence, the polymerization rate was strongly increased for all NHC-boranes (Fig. 4). In contrast to $\mathrm{BP} / \mathrm{EDB} / \mathrm{Ph}_{2} \mathrm{I}^{+} \mathrm{PF}_{6}{ }^{-}$ formulations, in which the amine component can react with the iodonium salt, the $\mathrm{BP} / \mathrm{NHC}$-borane/ $\mathrm{Ph}_{2} \mathrm{I}^{+} \quad \mathrm{PF}_{6}^{-}$formulations are stable for more than one week at rt.

\section{Conclusion}

NHC-boranes are a class of excellent co-initiators for free radical photopolymerization of acrylates. They are attractive for practical applications because they are compatible with both air and water thanks to the properties of boron radicals. The strength of the carbene-boron bond and the stability of the NHC-boranes are the keys to their use as reagents first in molecular chemistry, and now also in polymer synthesis. We believe this can only further develop in the near future.

\section{Acknowledgements}

We warmly thank the colleagues, students and postdoctoral co-workers, who participated in the polymer project, for their contributions. Special thanks go to Mohamad-Ali Tehfe, who carried out most of the polymerizations. Our work was supported by grants from the US National Science Foundation (CHE-0645998), UPMC, IUF, CNRS, and the French Agence Nationale de la Recherche (ANR, BLAN0309 Radicaux Verts, and 08-CEXC-011-01 Borane). D. P. C. thanks l'État et la Région Île de France for a 'Chaire Blaise Pascal'. E. L. thanks Actelion and ENSCMu for the Actelion award.

Received: March 6, 2012 


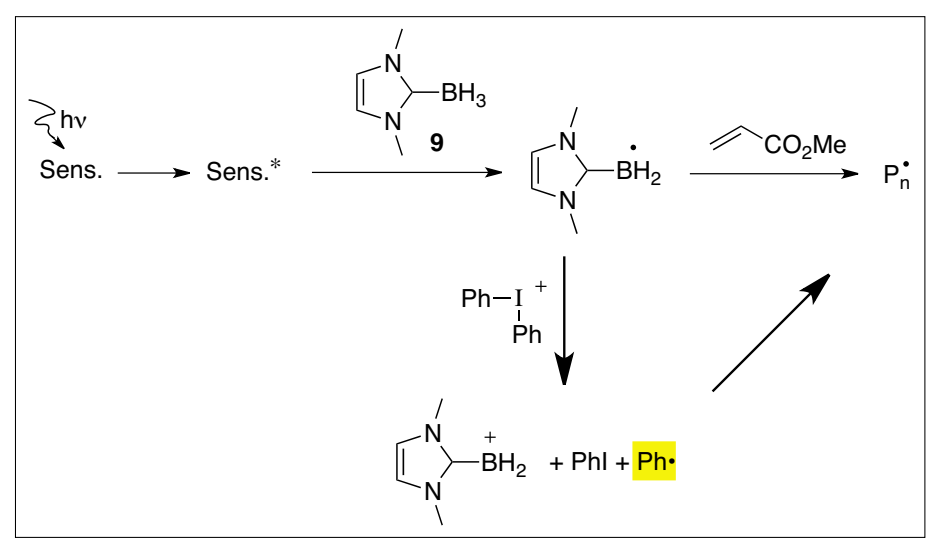

Scheme 4. Acceleration of the polymerization with diphenyliodonium salts.

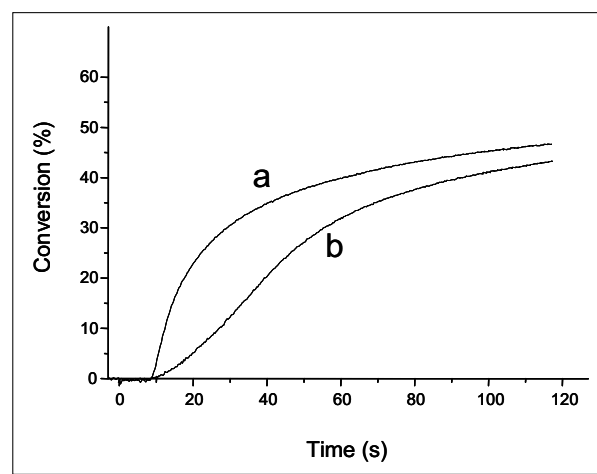

Fig. 4. Influence of diphenyliodonium on the radical photopolymerization of TMPTA: (a) $\mathrm{BP} / 2 / \mathrm{Ph}_{2} \mathrm{I}^{+}(1 \% / 1 \% / 1 \% \mathrm{w} / \mathrm{w}) ;(\mathrm{b}) \mathrm{BP} / 2$ $(1 \% / 1 \% \mathrm{w} / \mathrm{w})$.

[4] M.-A. Tehfe, J. Monot, M. Brahmi, H. Bonin-Dubarle, D. P. Curran, M. Malacria L. Fensterbank, E. Lacôte, J. Lalevée, J. P. Fouassier, Polym. Chem. 2011, 2, 625.

[5] M.-A. Tehfe, M. Makhlouf Brahmi, J. P. Fouassier, D. P. Curran, M. Malacria, L. Fensterbank, E. Lacôte, J. Lalevée, Macromolecules 2010, 43, 2261.

[6] S.-H. Ueng, L. Fensterbank, E. Lacôte, M. Malacria, D. P. Curran, Org. Lett. 2010, 12, 3002.

[7] M.-A. Tehfe, J. Monot, M. Malacria, L. Fensterbank, J. P. Fouassier, D. P. Curran, E. Lacôte, J. Lalevée, ACS Macro Lett. 2012, 1, 92.

[8] T. Matsumoto, F. P. Gabbaï, Organometallics 2009, 28, 4252 . 